\title{
Red Rain Cells of Kerala as a Possible Carrier of the Diffuse Interstellar Bands and the UV Extinction Bump
}

\author{
A. Santhosh Kumar ${ }^{1}$, N. Chandra Wickramasinghe ${ }^{2,3,4}$ and Godfrey Louis ${ }^{{ }^{*}}$ \\ ${ }^{1}$ School of Pure \& Applied Physics, Mahatma Gandhi University, Priyadarshini Hills P.O, Kottayam-686560, \\ Kerala, India. \\ ${ }^{2}$ Centre for Astrobiology, The University of Buckingham, Buckingham, MK18 1EG, UK. \\ ${ }^{3}$ Institute for the Study of Panspermia and Astroeconomics, Gifu, Japan. \\ ${ }^{4}$ Centre for Astrobiology, University of Ruhuna, Matara, Sri Lanka. \\ ${ }^{5}$ Astrobiology Division, Department of Physics, Cochin University of Science \& Technology, Cochin-682022, \\ Kerala, India. \\ Email: godfrey.louis@gmail.com
}

\begin{abstract}
Despite various proposals, no conclusive identification exists for the carriers of diffuse interstellar absorption bands DIBs and the UV extinction bump at $217.5 \mathrm{~nm}$. The red rain cells of Kerala show strong absorption features near $216.5 \mathrm{~nm}$ and several weak absorption features in the wavelength region 400 to $900 \mathrm{~nm}$ which are superimposed on a broader blue-UV absorption peak. The red pigment extracted from the cells show strong peaks at $334 \mathrm{~nm}$ and $440 \mathrm{~nm}$ along with multitude of weak peaks. The measured absorption peaks are compared with that of DIBs and the UV bump. The higher peak widths for red cells in comparison with DIBs is attributed to the temperature difference between space environment and laboratory. Our results of the absorption spectra of red rain cells show significant correlation with the reported wavelengths of numerous DIBs and the UV extinction bump of the interstellar medium, thus showing that the red rain cells, which are of suspected extraterrestrial origin, is a plausible carrier of DIBs and the UV extinction bump.
\end{abstract}

Keywords: Diffuse interstellar bands, UV extinction bump, interstellar dust, red rain of Kerala

\section{Introduction}

The Diffuse Interstellar Bands (DIBs) and the ultraviolet extinction bump at $2175 \AA$ are two unresolved spectroscopic puzzles of the interstellar medium (ISM). The other two mysterious spectroscopic features of the ISM are Extended Red Emission (ERE) and Unidentified Infrared bands (UIR). The DIBs are absorption lines detected in the spectra of stars that are obscured by interstellar clouds [1]. They are too broad to be associated with atomic lines and are observed in a spectral range extending from $4000 \AA$ to $10000 \AA$ exhibiting a strong diversity of profiles [2].

The identification of the carriers of the DIBs is one of the most challenging problems in astrophysics since the discovery of the first two such features [3]. The survey of Herbig [4] reported 39 DIBs. Since that time, the number of DIBs has been extended to more than $220[5,6]$ and more than 250 DIBs are reported by Tuairisg et al. [7] with the highest density of bands found in the visible region (5400 - 6900 $\AA$ ) $[2,6]$. Further, over 400 DIBs have been observed in the visible and near-infrared wavelength range in both the Milky Way and external galaxies, both nearby and distant [8]. The individual bands exhibit a wide variety of strengths and widths (full width at half maximum (FWHM) values for the DIBs range from about 0.6 to $40.0 \AA$ ).

A large number of surveys were initiated to understand the nature of the DIB carriers. It is now generally accepted that the DIBs are most likely due to free molecules in the gas phase [2], but so far there has been no definitive match between a subset of the diffuse bands and the gas-phase spectrum of any individual molecule. In a recent study, two DIBs were provisionally identified as due to fullerene $\mathrm{C}_{60}{ }^{+}$ [9]. The current consensus is that the DIB carriers are most probably complex organic molecules in the neutral and/or ionized forms [10]. It has been suggested by many authors that these peaks arise from PAH structures, but objections raised initially by Schlemmer et al. [11] would still appear to be largely 
valid. Moreover the formation of PAHs abiotically also poses problems [12], particularly because abiotic processes would not be expected to lead to the observed invariance of the characteristics of these bands.

The origin of the $217.5 \mathrm{~nm}$ absorption feature appearing in the interstellar extinction curve is a long-standing problem in astrophysics [13]. It is characterized by a stable position, but its band width changes according to the interstellar environment [14]. Many types of carbon and carbonaceous materials have been proposed to explain the $217 \mathrm{~nm}$ feature, including graphite [15], graphite spheres [16,17], hydrogenated amorphous carbon (HAC) [18], coal-like material [19], fullerenes [20], graphite onions [21]. An important contribution of a mixture of PAHs to the UV bump has been proposed by Joblin et al. [22]. Among the other proposed candidates are particles composed of silicate or graphite cores with a mantle of PAHs [23] and carbon onion particles [24,20]. All recent results point strongly toward a carbonaceous carrier for the UV bump. Alternative molecular explanations of the $2175 \AA$ absorption, first proposed by Hoyle and Wickramasinghe in 1977, involve aromatic organic molecules [25]. The interstellar absorption feature at $2175 \AA$ in stellar spectra could possibly be attributed to aromatic molecules within biological cells $[12]$.

Here we show that the red cells which caused the red colour of the red rain of Kerala in 2001 are a possible candidate as a carrier of the DIBs and the UV extinction bump in the interstellar medium. The UV-visible-NIR absorption spectra of the red rain cells and its extracted red pigment in the range 190 to $900 \mathrm{~nm}$ can also be correlated with the spectra of DIBs and the UV extinction bump of ISM.

\subsection{Evidences for Unusual Nature and Extraterrestrial Origin of Red Rain Cells}

In an earlier paper we have argued that the red cells that caused the red rain of Kerala in 2001 are possibly of extraterrestrial origin [26]. These cells were proposed to have originated from fragments of a cometary meteor, which disintegrated in the upper atmosphere and it was argued that the red rain phenomenon is a possible case of Cometary Panspermia. At least 50,000 kg of these cells were estimated to have fallen in Kerala during July to September 2001 through various isolated red rain events which occurred at places separated by geographical distances of more than $400 \mathrm{~km}$. It was also argued on the basis of spectro-fluorimetric study that the cells may not have DNA.

There are several cases of red rain events recorded in history which occurred in different parts of the world and it has been pointed out that many of these events have a correlation with meteoric falls [27]. In another study it was reported that IR spectrum of the red rain cells of Kerala was showing correlation with several unidentified IR emission bands of proto-planetary nebulae and it was commented that the cells could represent a completely unknown microorganism [28]. A study of the fluorescence properties of the cultured cells of these exotic microbes showed that they have an unusual kind of autofluorescence [29] which was interpreted as due to the possible presence of unidentified biomolecules. In a recent study we have argued that the red cells that caused the red rain of Kerala cannot be identified with Trentepohlia algal cells, because there are marked differences between red cells and Trentepohlia cells [30].

Reports of DNA detection using DAPI staining by Microscopic Probe technique after pigment removal by DMSO showed positive results [31]. Later we argued that the result of DAPI staining is not conclusive because of the continuous auto-fluorescence of the red cells, when its outer red pigment was removed [32]. The wide auto fluorescence behavior of the depigmented red cells has been described by Louis \& Kumar [32] and Gangappa \& Hogg [31]. Hence the blue fluorescence is not due to DNA rather due to autofluorescence of the red cells.

Using transmission electron microscopy, Gangappa et al. [33] demonstrated significant differences in the ultra-structure of red cells when compared with representative species from these groups. The red rain cells showed no evidence of typical eukaryotic internal structures such as mitochondria or endoplasmic reticulum. Also comparisons based on elemental composition using energy-dispersive X-ray analysis, as well as Raman spectral signatures demonstrate significant dissimilarities in their molecular composition and concluded that the identity and origins of the red rain cells remain an enigma [33].

Several episodes of red rain were also reported in the Central and Southern Provinces of Sri Lanka during late November and December 2012 [34]. The cause of the red coloration to the Sri Lankan red rain was due to huge quantity of red cells that had identical appearance to Kerala red rain cells. TEM and EDAX studies of the Sri Lankan red rain cells showed the outer cell wall unusually rich in uranium, and a nuclear region with a strong deficit or absence of phosphorus [34]. As phosphorus is present in DNA 
structure as phosphate molecule, the absence of phosphorus in the cell interior can be suggestive evidence that the cell does not have DNA.

\section{Experiments and Results}

\subsection{Broad Absorption Features of Red Rain Cells}

In our previous study [26] we have reported the absorption spectra of the red cells in the uv-visible region which showed major absorption peaks at $200 \mathrm{~nm}, 505 \mathrm{~nm}$ and at $600 \mathrm{~nm}$. Here the absorption spectra in the visible and near IR region have been analyzed more rigorously. The results of the UV-Visible absorption spectra of the red rain cells in the range 190 to $900 \mathrm{~nm}$ is shown in Fig. 1 for different red rainwater samples collected from various locations.

The concentrations of red cells suspended in the rainwater collected from different locations vary from sample to sample. Red cells collected from location 1 showed an absorption peak at $214.5 \mathrm{~nm}$ whereas the sample collected from location 2 showed an absorption peak at $213 \mathrm{~nm}$ and the sample collected from location 3 shows an absorption peak at $216.5 \mathrm{~nm}$. This minor shift in the intensity peak is due to the red cells concentration. To study the concentration dependent absorption peak in the UV region, spectra were also recorded for diluted samples. The resultant spectra showed a peak shift towards lower wavelength region as concentration decreases. This result definitely does not fit with the absorption of most substances in solution, where a decrease in the absorption intensity is obtained as the concentration of the substance decreases. This contrary result observed in the red rain cells may be partly due to the clustering of the red cells in the sample. Particle clustering may strongly influence the absorption characteristics of nano-sized particles [35]. For a sample with highly concentrated red cells, the absorption peak was observed at $216.5 \mathrm{~nm}$ (sample collected from location 3, as indicated in the plot). A wavelength shift of about $1 \mathrm{~nm}$ between the laboratory sample and the interstellar material may be explained as due to the lower temperature of interstellar grains.

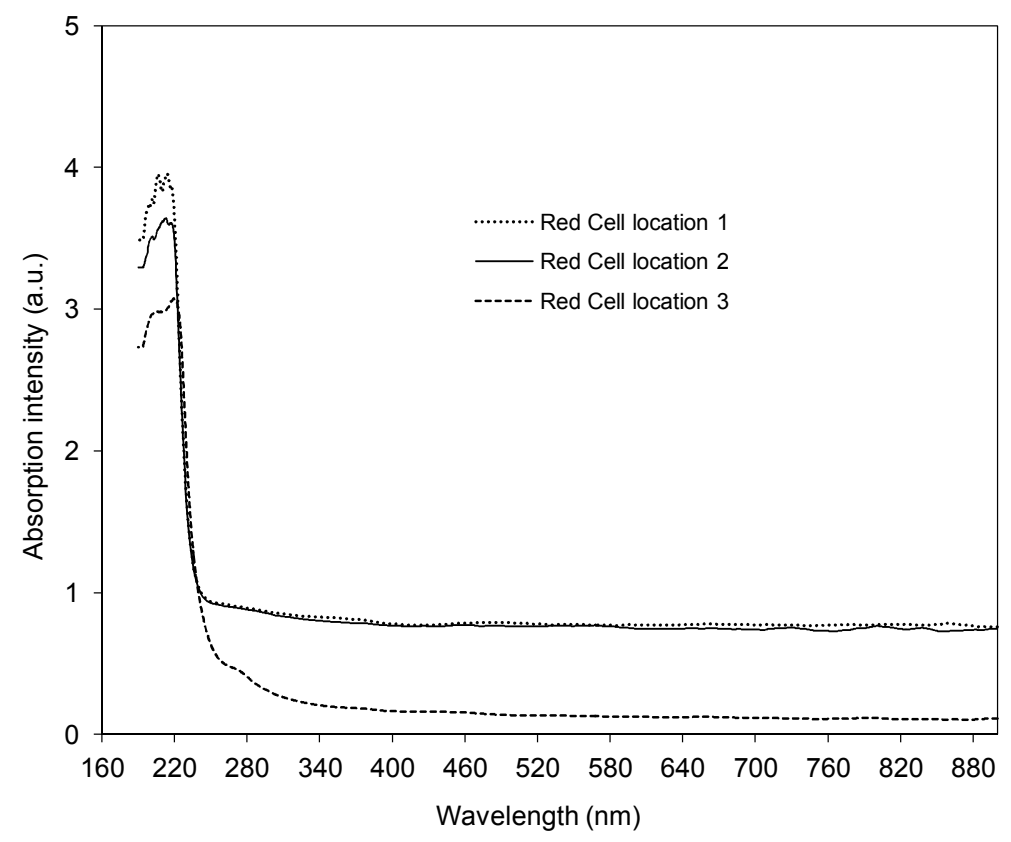

Figure 1: Absorption spectra of red cells for red rainwater samples collected from three different locations in Kerala, India during the 2001 red rain event. 


\subsection{Detailed Absorption Spectra of Red Rain Cells}

The absorption spectra of the red rainwater collected from various locations showed several weak narrow absorption features in the visible and near IR region which are superimposed on a broader absorption peak. Since the absorption spectra of red cells show a large number of small peaks, the spectra are presented in two separate plots. The plot Fig. 2 (a) shows the absorption spectra of red cells in the wavelength range from 400 to $700 \mathrm{~nm}$, whereas the plot Fig. 2 (b) shows the spectra in the region 700 to $900 \mathrm{~nm}$. Spectra of the red cells in the wavelength range $400-700 \mathrm{~nm}$ showed 20 absorption peaks in which the strongest profile peaks were observed at $484.5 \mathrm{~nm}, 539 \mathrm{~nm}$ and $664 \mathrm{~nm}$. In the wavelength region 700-900 $\mathrm{nm}$ the spectra of the sample shows another 20 peaks.
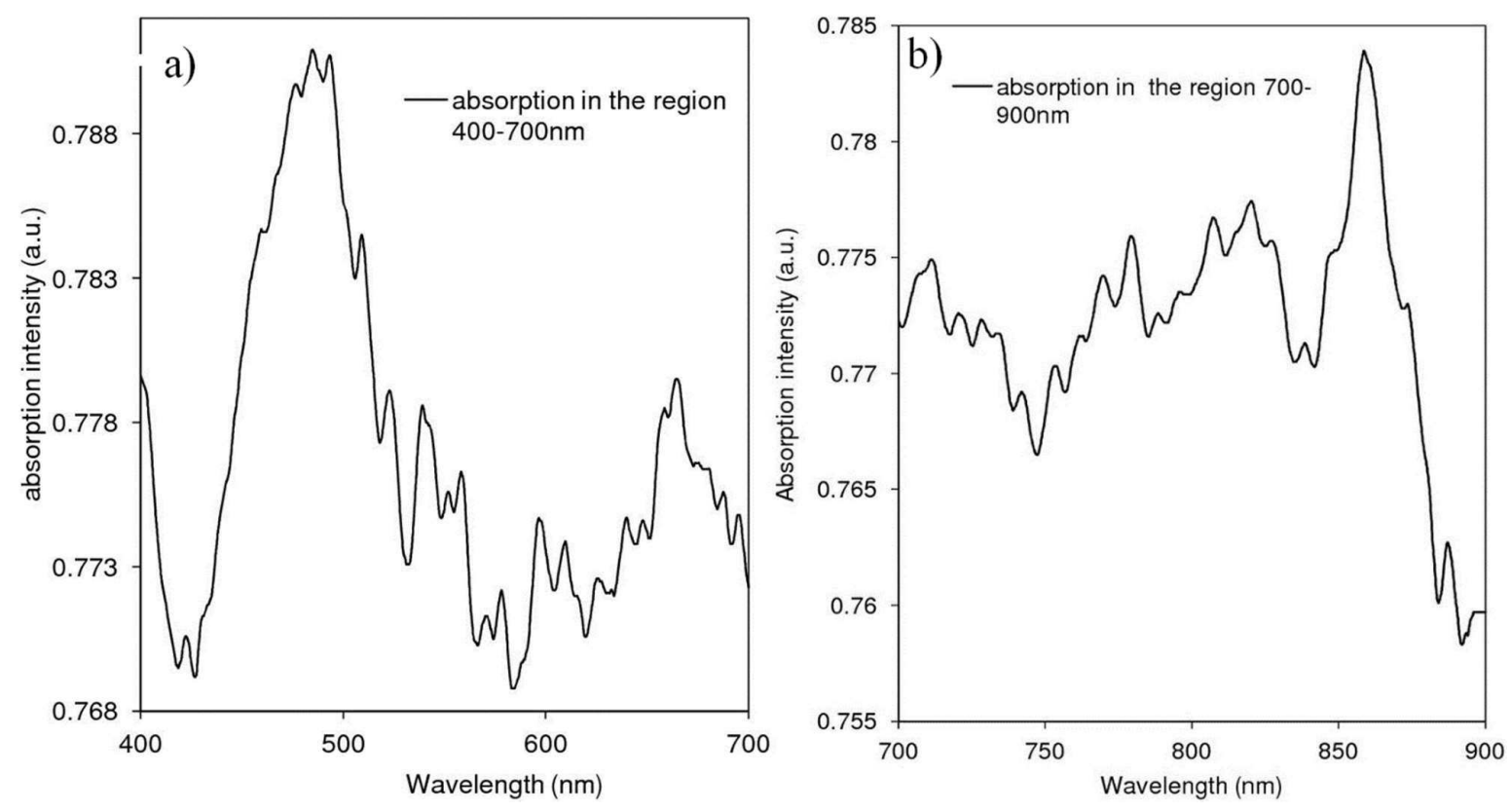

Figure 2: Detailed optical absorption spectra of the red rain cells of Kerala showing the weak absorption peaks : a) In the visible region from $400-700 \mathrm{~nm}$, b) In the near IR region from 700 - $900 \mathrm{~nm}$. Deconvoluted peaks are shown in Fig. 3. The measurements were made using UV Vis spectrophotometer (Jasco model V-650 and confirmed by Shimadzu spectrophotometer (model UV-2401 PC).

The spectra of red cell have been deconvoluted using a Gaussian deconvolution method and the resulting spectrum is shown in Fig. 3 for wavelength intervals of $100 \mathrm{~nm}$ (Fig. 3 (A)-(E)). The deconvoluted data are tabulated in Table 1 along with the peak position and corresponding bandwidth. The highest density of absorption peaks was observed in the region 500-700 $\mathrm{nm}$, the strongest absorption peaks being at $639 \mathrm{~nm}, 483 \mathrm{~nm}, 518 \mathrm{~nm}$ and $540 \mathrm{~nm}$. The other prominent peaks are observed at 826, 840,767 and at $679 \mathrm{~nm}$. The widths of these bands range from 16 to $24 \mathrm{~nm}$, which are a factor of 10 higher than the widths of the DIBs. This may seem problem for our model at first sight, but the problem could be resolved if we note that the laboratory temperatures are about 10 times higher than the temperature of interstellar grains. With regard to central wavelength many of these peaks can be correlated with DIBs of the ISM with a wavelength deviation much below $1 \mathrm{~nm}$. 

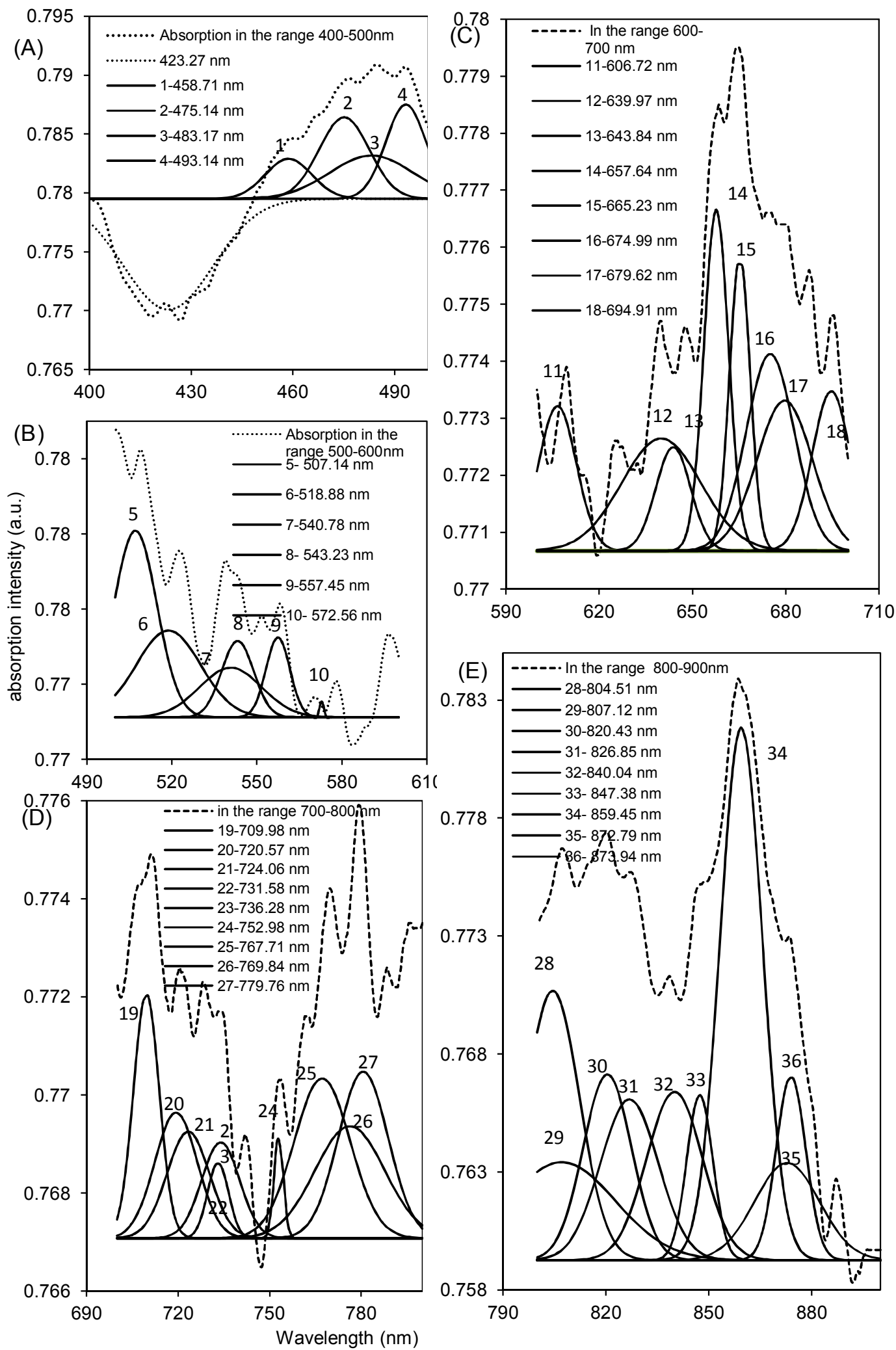

Figure 3: The absorption spectra of the red rain cells in the wavelength intervals of $100 \mathrm{~nm}$. The deconvoluted Gaussian peaks are shown in solid lines. (A) in the range $400-500 \mathrm{~nm}$, (B) in the range $500-600 \mathrm{~nm},(\mathrm{C})$ in the range $600-700 \mathrm{~nm},(\mathrm{D}) 700-800 \mathrm{~nm},(\mathrm{E}) 800-900 \mathrm{~nm}$. The deconvoluted spectra is showing large numbers of peaks. 36 peaks are observed which are labeled as 1 to 36 and given in the Table 1 along with the reported DIBs. Dotted lines represent the original spectrum. 


\subsection{Absorption Spectra of the Red Pigment Extracted from the Red Rain Cells}

The red pigment of the red rain cells was extracted from the cells by immersing in the solvent DMSO at $90^{\circ} \mathrm{C}$ for a period of 16 hours. The UV-Visible and near IR absorption spectra of the extracted red pigment were recorded. They clearly show broad and finer features. There is a distinct peak at $334 \mathrm{~nm}$, a broad peak centered at $440 \mathrm{~nm}$ and a shoulder peak at $558 \mathrm{~nm}$ as shown in Fig. 4. The spectrum also shows several weak absorption peaks as shown in Fig. 5 (a), Fig. 5(b) and Fig. 6 for the wavelength regions $600-700 \mathrm{~nm}, 700-800 \mathrm{~nm}$ and $800-900 \mathrm{~nm}$ respectively. These spectra registered 63 weak absorption peaks in the wavelength region $600-900 \mathrm{~nm}$ and are listed in Table 2 along with the reported DIBs. The spectrum does have features close to $4430 \AA$ which is closest to the strongest DIBs in the ISM. The peak near $216.5 \mathrm{~nm}$ in the red cells as a whole is absent in the extracted pigment whereas a high intensity peak at $334 \mathrm{~nm}$ was observed.

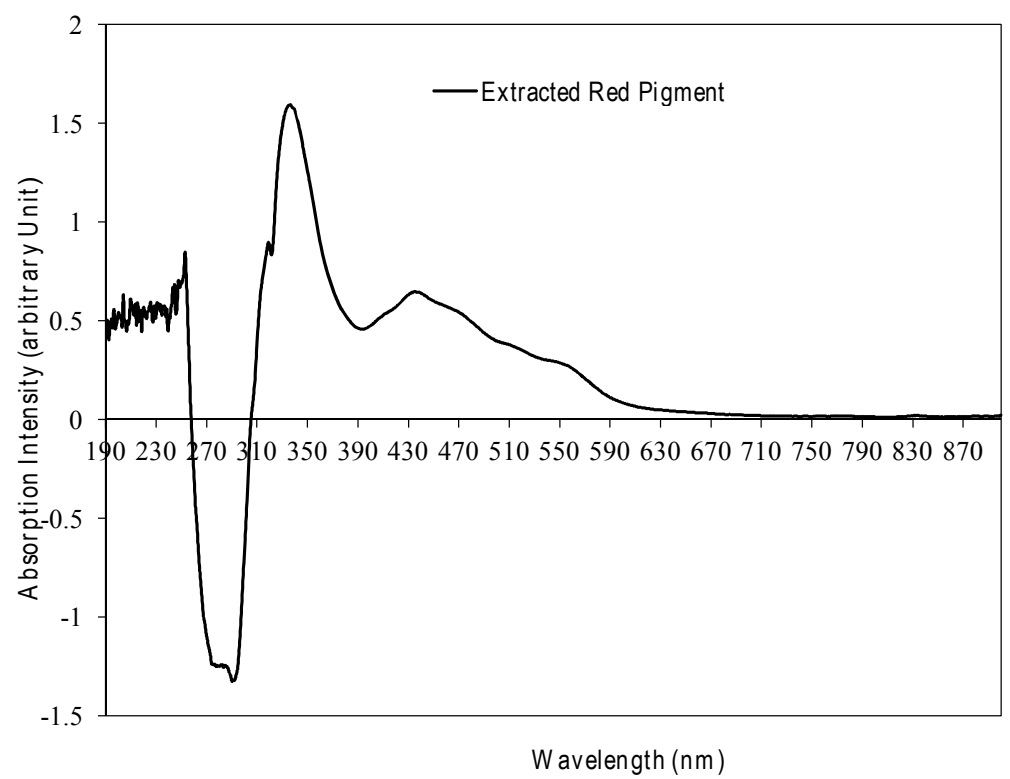

Figure 4: Broad absorption spectra of the red pigment extracted using DMSO from the red rain cells.
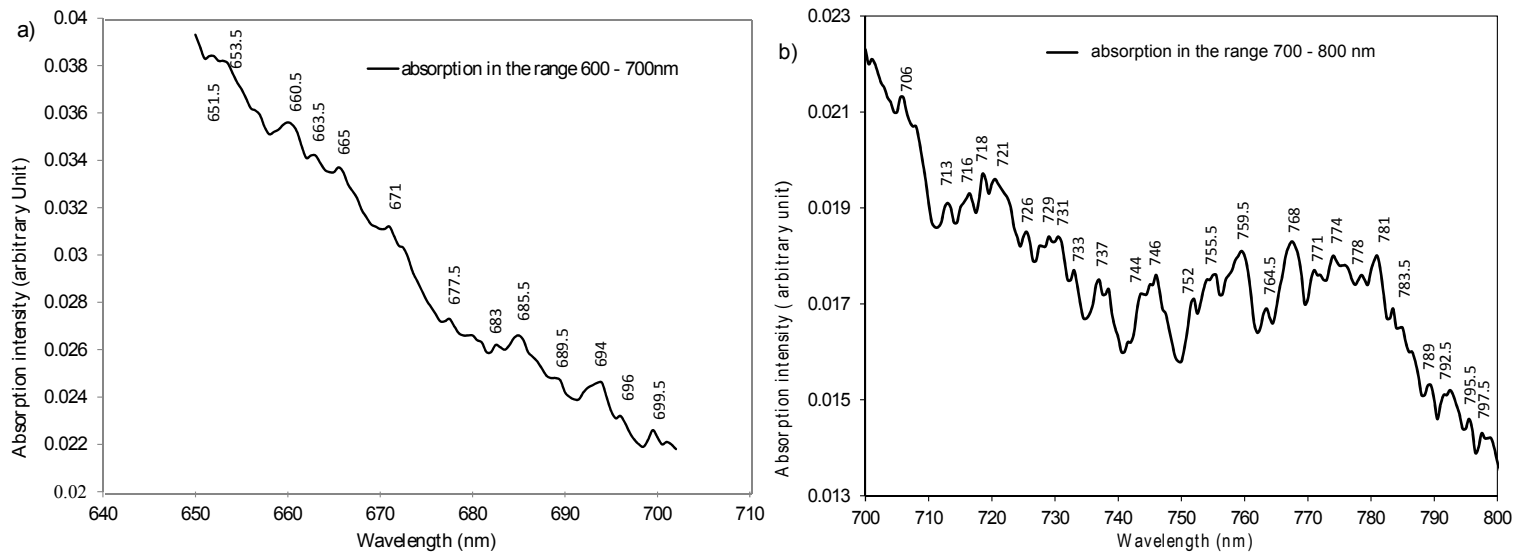

Figure 5: a) Absorption spectra of the extracted red pigment in the wavelength region $600-700 \mathrm{~nm}$ which registered 13 peaks b) Absorption spectra of the extracted red pigment in the wavelength region $700-800 \mathrm{~nm}$ which registered 26 peaks. These are listed and compared with DIBs in Table 2. 


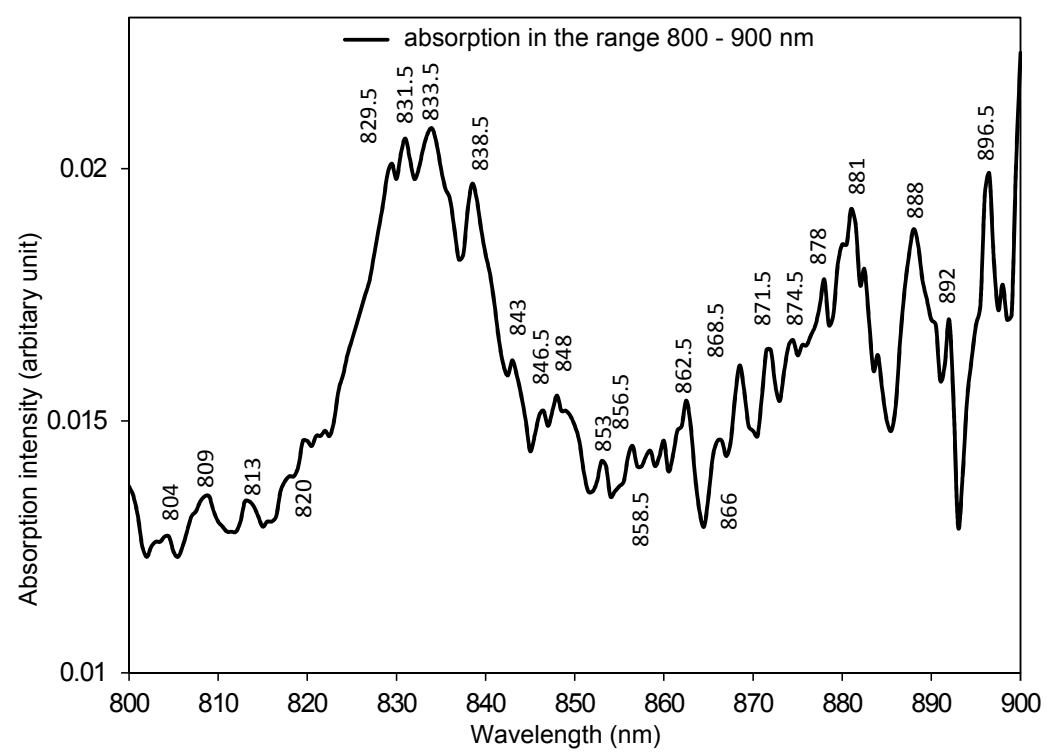

Figure 6: Absorption spectra of the extracted red pigment in the wavelength region 800-900 $\mathrm{nm}$ which registered 24 peaks. These peaks are listed and compared with DIBs in Table 2.

Table 1: Observed Absorption peaks of red rain cells compared with reported DIBs in ISM

\begin{tabular}{|c|c|c|c|c|c|c|c|c|c|}
\hline $\begin{array}{l}\text { Peak } \\
\text { No. }\end{array}$ & $\begin{array}{l}\text { Observed } \\
\text { Peaks } \\
n m\end{array}$ & $\begin{array}{l}\text { FWHM } \\
n m\end{array}$ & $\begin{array}{l}\text { Reported } \\
\text { DIBs } \\
\AA\end{array}$ & $\begin{array}{l}\text { Devi- } \\
\text { ation } \\
\mathrm{nm}\end{array}$ & $\begin{array}{l}\text { Peak } \\
\text { No. }\end{array}$ & $\begin{array}{l}\text { Observed } \\
\text { peaks } \\
n m\end{array}$ & $\begin{array}{l}\text { FWHM } \\
n m\end{array}$ & $\begin{array}{l}\text { Reported } \\
\text { DIBs } \\
\AA\end{array}$ & $\begin{array}{l}\text { Devi- } \\
\text { ation } \\
n m\end{array}$ \\
\hline 1. & 458.71 & 13.737 & 4593.93 & -0.68 & 19. & 709.98 & 8.1545 & 7099.48 & +0.03 \\
\hline 2. & 475.14 & 14.834 & 4762.57 & -1.12 & 20. & 720.57 & 12.510 & 7223.13 & -1.74 \\
\hline 3. & 483.17 & 24.540 & 4825.94 & +0.58 & 21. & 724.06 & 13.431 & 7236.36 & +0.42 \\
\hline 4. & 493.34 & 11.877 & 4951.05 & -1.77 & 22. & 731.58 & 5.2548 & 7321.08 & -0.53 \\
\hline 5. & 507.14 & 15.179 & 5061.56 & +0.98 & 23. & 736.28 & 10.680 & 7360.45 & +0.24 \\
\hline 6. & 518.82 & 22.671 & 5176.00 & +1.22 & 24. & 752.98 & 3.6058 & 7558.5 & -2.87 \\
\hline 7. & 540.78 & 21.965 & 5404.52 & +0.33 & 25. & 767.71 & 17.469 & 7686.46 & -0.94 \\
\hline 8. & 543.23 & 11.283 & 5419.05 & +1.33 & 26. & 769.84 & 14.102 & 7695.92 & +0.25 \\
\hline 9. & 557.45 & 8.4210 & 5569.08 & +0.54 & 27. & 779.76 & 5.4460 & 7782.25 & +1.53 \\
\hline 10. & 572.56 & 0.7748 & 5719.68 & +0.59 & 28. & 804.51 & 15.553 & 8038.48 & +0.66 \\
\hline 11. & 606.72 & 15.357 & 6068.45 & -0.13 & 29. & 807.12 & 31.269 & 8040.08 & +3.11 \\
\hline 12. & 639.97 & 25.355 & 6400.37 & -0.07 & 30. & 820.43 & 14.128 & & \\
\hline 13. & 643.84 & 11.194 & 6439.42 & +0.10 & 31. & 826.85 & 16.794 & 8283.45 & -1.5 \\
\hline 14. & 657.64 & 7.8575 & 6591.4 & -1.5 & 32. & 840.04 & 16.323 & & \\
\hline 15. & 665.23 & 6.5904 & 6645.95 & +0.63 & 33. & 847.38 & 7.6525 & & \\
\hline 16. & 674.99 & 15.499 & 6740.96 & +0.89 & 34. & 859.45 & 12.289 & 8621.11 & -2.66 \\
\hline 17. & 679.62 & 17.901 & 6795.18 & +0.1 & 35. & 872.79 & 18.900 & & \\
\hline 18. & 694.61 & 12.292 & 6944.53 & +0.16 & 36. & 873.94 & 8.6165 & & \\
\hline
\end{tabular}


Table 2: Observed absorption peaks of extracted pigment of the red rain cells compared with reported DIBs in ISM

\begin{tabular}{|c|c|c|c|c|c|c|c|}
\hline No. & $\begin{array}{l}\text { Observed } \\
\text { peaks } \\
\mathrm{nm}\end{array}$ & $\begin{array}{l}\text { Reported } \\
\text { DIBs } \\
\AA\end{array}$ & $\begin{array}{l}\text { Deviation } \\
\mathrm{nm}\end{array}$ & No. & $\begin{array}{l}\text { Observed } \\
\text { peaks } \\
\mathrm{nm}\end{array}$ & $\begin{array}{l}\text { Reported } \\
\text { DIBs } \\
\AA\end{array}$ & $\begin{array}{l}\text { Deviation } \\
\mathrm{nm}\end{array}$ \\
\hline 1 & 334 & 3340.00 & 0.00 & 36 & 764.5 & 7651.37 & -0.637 \\
\hline 2 & 440 & 4430.00 & -3 & 37 & 768 & 7686.46 & -0.646 \\
\hline 3 & 463 & 4666.65 & -3.665 & 38 & 771 & 7709.67 & +0.033 \\
\hline 4 & 509 & 5110.89 & -2.089 & 39 & 774 & 7748.18 & -0.818 \\
\hline 5 & 558 & 5569.08 & +1.092 & 40 & 778 & 7782.25 & -0.225 \\
\hline 6 & 615 & 6151.15 & -0.115 & 41 & 781 & & \\
\hline 7 & 618 & 6185.89 & -0.589 & 42 & 783.5 & 7832.72 & +0.228 \\
\hline 8 & 651.5 & 6520.70 & -0.57 & 43 & 789 & 7904.92 & -1.492 \\
\hline 9 & 653.5 & 6536.44 & -0.144 & 44 & 792.5 & 7927.80 & -0.28 \\
\hline 10 & 660.5 & 6613.72 & -0.872 & 45 & 795.5 & 7935.33 & +1.967 \\
\hline 11 & 663.5 & 6632.93 & +0.207 & 46 & 797.5 & 7987.89 & -1.289 \\
\hline 12 & 665 & 6660.64 & -1.064 & 47 & 804 & 8038.48 & +0.152 \\
\hline 13 & 671 & 6709.24 & +0.076 & 48 & 809 & & \\
\hline 14 & 677.5 & 6770.05 & +0.495 & 49 & 813 & & \\
\hline 15 & 683 & 6827.28 & +0.272 & 50 & 820 & & \\
\hline 16 & 685.5 & 6852.90 & +0.21 & 51 & 829.5 & 8283.45 & +1.155 \\
\hline 17 & 689.5 & 6886.92 & +0.808 & 52 & 831.5 & & \\
\hline 18 & 694 & 6944.00 & -0.400 & 53 & 833.5 & & \\
\hline 19 & 696 & 6978.54 & -1.854 & 54 & 838.5 & & \\
\hline 20 & 699.5 & 6998.71 & -0.371 & 55 & 843 & & \\
\hline 21 & 706 & 7060.81 & -0.081 & 56 & 846.5 & & \\
\hline 22 & 713 & 7136.09 & -0.609 & 57 & 848 & & \\
\hline 23 & 716 & 7161.21 & -0.121 & 58 & 853 & & \\
\hline 24 & 718 & 7179.95 & +0.005 & 59 & 856.5 & & \\
\hline 25 & 721 & 7223.13 & -1.313 & 60 & 858.5 & 8530.79 & +5.421 \\
\hline 26 & 726 & 7264.98 & -0.498 & 61 & 862.5 & 8621.23 & +0.377 \\
\hline 27 & 729 & 7287.62 & +0.238 & 62 & 866 & 8648.28 & +1.172 \\
\hline 28 & 731 & 7321.08 & -1.108 & 63 & 868.5 & & \\
\hline 29 & 733 & 7330.17 & -0.017 & 64 & 871.5 & & \\
\hline 30 & 737 & 7369.29 & +0.071 & 65 & 874.5 & & \\
\hline 31 & 744 & 7432.07 & +0.793 & 66 & 878 & & \\
\hline 32 & 746 & & & 67 & 881 & & \\
\hline 33 & 752 & & & 68 & 888 & & \\
\hline 34 & 755.5 & 7558.50 & -0.35 & 69 & 892 & & \\
\hline 35 & 759.5 & 7585.63 & +0.937 & 70 & 896.5 & & \\
\hline
\end{tabular}




\section{Concluding Remarks}

Our investigation of the optical properties of the red rain cells showed a significant correlation with well-known interstellar extinction bump at $217.5 \mathrm{~nm}$. Furthermore, the red rain cells exhibit many correspondences with the DIB's in the wavelength range 400 - $900 \mathrm{~nm}$. In particular, the pigment extracted from the red cells showed a broad absorption peak centered on $440 \mathrm{~nm}$ and a high intensity peak at $334 \mathrm{~nm}$. The density of absorption peaks was higher in the wavelength region $550-700 \mathrm{~nm}$, similar to the situation for DIBs of the ISM. In conclusion we note that the interstellar absorption feature at $217.5 \mathrm{~nm}$ and the DIBs of the ISM are plausibly attributed to the presence of the red cells in the ISM. The red cells have unusual autofluorescence and a broad emission peak in the wavelength range 520 - 720 $\mathrm{nm}$ which can be correlated with ERE of the ISM and planetary nebulae. It has been reported elsewhere that the IR spectrum of the red rain cells of Kerala showed a correlation with several unidentified IR emission bands of protoplanetary nebulae [28].

The present identification of a correlation between the optical absorption features of red rain cells and its extracted pigment and the observed optical absorption features of interstellar medium is, in our view, highly significant. In conclusion we note that these results support the view that the red rain cells of both Kerala (2001) and Sri Lanka (2012) may have an extraterrestrial origin with an ubiquitous presence throughout the universe.

\section{References}

1. P. Ehrenfreund and S. B. Charnley, "Organic Molecules in the Interstellar Medium, Comets, and Meteorites: A Voyage from Dark Clouds to the Early Earth," Annual Review of Astronomy and Astrophysics, vol. 38, no. 1, pp. 427-483, 2000. [Online]. Available: https://doi.org/10.1146/annurev.astro.38.1.427

2. G. Herbig, "The Diffuse Interstellar Bands," Annual Review of Astronomy and Astrophysics, vol. 33, pp. 19-74, 1995.

3. M. Heger, "The spectra of certain class B stars in the regions 5630A-6680A and 3280A-3380A," Lick Observatory Bulletin, vol. 10, pp. 146-147, 1922.

4. G. Herbig, "The diffuse interstellar bands. IV - The region 4400-6850 A," Ap. J., vol. 196, pp. 129-160, feb 1975.

5. F. Salama, G. Galazutdinov, J. Krełowski, L. Allamandola, and F. Musaev, "Polycyclic Aromatic Hydrocarbons and the Diffuse Interstellar Bands: A Survey," The Astrophys. Journ., vol. 526, pp. 265-273, nov 1999.

6. P. Jenniskens and F.-X. Desert, "A survey of diffuse interstellar bands (3800-8680 A)," Astronomy and Astrophysics Suppl., vol. 106, pp. 39-78, jul 1994.

7. S. Ó. Tuairisg, J. Cami, B. H. Foing, P. Sonnentrucker, and P. Ehrenfreund, "A deep echelle survey and new analysis of diffuse interstellar bands ," Astron. Astrophys. Suppl. Ser., vol. 142, no. 2, pp. 225-238, 2000. [Online]. Available: https://doi.org/10.1051/aas:2000148

8. F. Y. Xiang, A. Li, and J. X. Zhong, "A tale of two mysteries in interstellar astrophysics: the $2175 \AA$ extinction bump and diffuse interstellar bands," The Astrophysical Journal, vol. 733, no. 2, p. 91, may 2011. [Online]. Available: https://doi.org/10.1088\%2F0004-637x\%2F733\%2F2\%2F91

9. E. K. Campbell, M. Holz, D. Gerlich, and J. P. Maier, "Laboratory confirmation of $\mathrm{C}_{60}^{+}$as the carrier of two diffuse interstellar bands," Nature, vol. 523, p. 322, jul 2015. [Online]. Available: https://doi.org/10.1038/nature14566

10. A. Tielens and T. Snow, The Diffuse Interstellar Bands. Springer Netherlands, 1995.

11. S. Schlemmer, D. J. Cook, J. A. Harrison, B. Wurfel, W. Chapman, and R. J. Saykally, "The unidentified interstellar infrared bands: PAHs as carriers?" Science, vol. 265, no. 5179, pp. 1686-1689, 1994. [Online]. Available: http://science.sciencemag.org/content/265/5179/1686

12. K. Rauf and C. Wickramasinghe, "Evidence for biodegradation products in the interstellar medium," International Journal of Astrobiology, vol. 9, no. 1, pp. 29-34, 2010.

13. B. T. Draine, "On the Interpretation of the $\lambda 2175 \AA$ Feature," in Interstellar Dust, ser. IAU Symposium, L. Allamandola and A. Tielens, Eds., vol. 135. Springer Netherlands, 1989, p. 313.

14. E. Fitzpatrick and D. Massa, "An analysis on the shapes of ultraviolet extinction curves. I - The $2175 \AA$ bump," Astrophysical Journal, vol. 307, pp. 286-294, aug 1986.

15. B. Draine and H. Lee, "Optical properties of interstellar graphite and silicate grains," Astrophysical Journal, vol. 285 , pp. 89-108, oct 1984.

16. F. Hoyle and N. Wickramasinghe, "On graphite particles as interstellar grains," Monthly Notices of the Royal Astronomical Society, vol. 124, p. 417, 1962. 
17. B. T. Draine, "Interstellar Dust Grains," Annual Review of Astronomy and Astrophysics, vol. 41, no. 1, pp. 241-289, 2003. [Online]. Available: https://doi.org/10.1146/annurev.astro.41.011802.094840

18. W. Duley, "Refractive indices for amorphous carbon," Astrophysical Journal, vol. 287, pp. 694-696, dec 1984.

19. R. Papoular, J. Conard, O. Guillois, I. Nenner, C. Reynaud, and J.-N. Rouzaud, "A comparison of solid-state carbonaceous models of cosmic dust." Astronomy and Astrophysics, vol. 315, pp. 222-236, nov 1996.

20. W. Krätschmer, L. D. Lamb, K. Fostiropoulos, and D. R. Huffman, "Solid C60: a new form of carbon," Nature, vol. 347, no. 6291, pp. 354-358, 1990. [Online]. Available: https://doi.org/10.1038/347354a0

21. W. A. de Heer and D. Ugarte, "Carbon onions produced by heat treatment of carbon soot and their relation to the $217.5 \mathrm{~nm}$ interstellar absorption feature," Chemical Physics Letters, vol. 207, no. 4, pp. 480-486, 1993. [Online]. Available: http://www.sciencedirect.com/science/article/pii/000926149389033E

22. C. Joblin, A. Leger, and P. Martin, "Contribution of polycyclic aromatic hydrocarbon molecules to the interstellar extinction curve," Astrophysical Journal,Letters, vol. 393, pp. L79-L82, jul 1992.

23. J. Mathis, "The origin of variations in the 2175 A extinction bump," Astrophysical Journal, vol. 422, pp. 176-186, feb 1994.

24. S. Wada, C. Kaito, S. Kimura, H. Ono, and A. Tokunaga, "Carbonaceous onion-like particles as a component of interstellar dust," Astronomy and Astrophysics, vol. 345, pp. 259-264, may 1999.

25. F. Hoyle and N. Wickramasinghe, "Identification of the 2,200 $\AA$ interstellar absorption feature," Nature, vol. 270, p. 323, nov 1977.

26. G. Louis and A. S. Kumar, "The Red Rain Phenomenon of Kerala and its Possible Extraterrestrial Origin," Astrophysics and Space Science, vol. 302, no. 1-4, pp. 175-187, apr 2006. [Online]. Available: http://www.springerlink.com/content/dx713q4194v6r76t/

27. P. McCafferty, "Bloody rain again! Red rain and meteors in history and myth," International Journal of Astrobiology, vol. 7, no. 01, pp. 9-15, jan 2008. [Online]. Available: http://journals.cambridge.org/abstract_ S1473550407003904

28. K. Rauf, R. Gangappa, M. Thomas, C. Wickramasinghe, and A. Campbell, "Red rain cell research: A new perspective for interplanetary transfer of life," in European Planetary Science Congress 2009 Abstracts vol.4, 2009, pp. 707-1. [Online]. Available: http://meetingorganizer.copernicus.org/EPSC2009/EPSC2009-707-1.pdf

29. G. Louis and A. S. Kumar, "Unusual autofluorescence characteristic of cultured red-rain cells," in Proc.SPIE, vol. 7097, aug 2008. [Online]. Available: https://doi.org/10.1117/12.794041

30. A. S. Kumar, N. C. Wickramasinghe, and G. Louis, "A comparative study of Trentepohlia and Red Rain cells," International Journal of Recent Scientific Research, vol. 4, no. 8, pp. 1205-1209, 2013. [Online]. Available: http://www.recentscientific.com/comparative-study-trentepohlia-and-red-rain-cells

31. R. Gangappa and S. I. Hogg, "DNA unmasked in the red rain cells of Kerala," Microbiology, vol. 159, no. 1, pp. 107-111, 2013. [Online]. Available: https://mic.microbiologyresearch.org/content/journal/micro/10.1099/ mic.0.062711-0

32. G. Louis and A. S. Kumar, "Autofluorescence characteristics of the red rain cells," in SPIE Optical Engineering + Applications, R. B. Hoover, G. V. Levin, A. Y. Rozanov, and N. C. Wickramasinghe, Eds. International Society for Optics and Photonics, sep 2013, pp. 88 650I-88650I-10. [Online]. Available: http://dx.doi.org/10.1117/12.2024366

33. R. Gangappa, M. J. Burchell, and S. I. Hogg, "Morphological and Molecular Analysis Calls for a Reappraisal of the Red Rain Cells of Kerala," Current Microbiology, vol. 68, no. 2, pp. 192-198, feb 2014. [Online]. Available: http://link.springer.com/10.1007/s00284-013-0464-9

34. N. Miyake, T. Matsui, J. Wallis, D. H. Wallis, A. Samaranayake, K. Wickramarathne, and N. C. Wickramasinghe, "Discovery of Uranium in Outer Coat of Sri Lankan Red Rain Cells," Journal of Cosmology, vol. 22, pp. $10042-10050,2013$.

35. M. Schnaiter, H. Mutschke, J. Dorschner, T. Henning, and F. Salama, "Matrix-isolated Nano-sized Carbon Grains as an Analog for the 217.5 Nanometer Feature Carrier," Astrophysical Journal, vol. 498, pp. 486-496, may 1998. 